\title{
Effect of Orthokeratology on myopia progression: twelve-year results of a retrospective cohort study
}

\author{
Yueh-Chang Lee ${ }^{1}$, Jen-Hung Wang ${ }^{2,3}$ and Cheng-Jen Chiu ${ }^{1,4^{*}}$ (D)
}

\begin{abstract}
Background: Several studies reported the efficacy of orthokeratology for myopia control. Somehow, there is limited publication with follow-up longer than 3 years. This study aims to research whether overnight orthokeratology influences the progression rate of the manifest refractive error of myopic children in a longer follow-up period (up to 12 years). And if changes in progression rate are found, to investigate the relationship between refractive changes and different baseline factors, including refraction error, wearing age and lens replacement frequency. In addition, this study collects long-term safety profile of overnight orthokeratology.

Methods: This is a retrospective study of sixty-six school-age children who received overnight orthokeratology correction between January 1998 and December 2013. Thirty-six subjects whose baseline age and refractive error matched with those in the orthokeratology group were selected to form control group. These subjects were followed up at least for 12 months. Manifest refractions, cycloplegic refractions, uncorrected and best-corrected visual acuities, power vector of astigmatism, corneal curvature, and lens replacement frequency were obtained for analysis.
\end{abstract}

Results: Data of 203 eyes were derived from 66 orthokeratology subjects (31 males and 35 females) and 36 control subjects (22 males and 14 females) enrolled in this study. Their wearing ages ranged from 7 years to 16 years (mean $\pm \mathrm{SE}, 11.72 \pm 0.18$ years). The follow-up time ranged from 1 year to 13 years (mean $\pm \mathrm{SE}, 6.32 \pm 0$. 15 years). At baseline, their myopia ranged from $-0.5 \mathrm{D}$ to $-8.0 \mathrm{D}$ (mean $\pm \mathrm{SE},-3.70 \pm 0.12 \mathrm{D}$ ), and astigmatism ranged from $0 \mathrm{D}$ to $-3.0 \mathrm{D}$ (mean $\pm \mathrm{SE},-0.55 \pm 0.05 \mathrm{D}$ ). Comparing with control group, orthokeratology group had a significantly $(p<0.001)$ lower trend of refractive error change during the follow-up periods. According to the analysis results of GEE model, greater power of astigmatism was found to be associated with increased change of refractive error during follow-up years.

Conclusions: Overnight orthokeratology was effective in slowing myopia progression over a twelve-year follow-up period and demonstrated a clinically acceptable safety profile. Initial higher astigmatism power was found to be associated with increased change of refractive error during follow-up years.

Keywords: Myopia, Myopia control, Orthokeratology, Contact lenses, Optical intervention

\footnotetext{
* Correspondence: drcjchiu@tzuchi.com.tw

1Department of Ophthalmology, Buddhist Tzu Chi General Hospital, No. 707,

Sec. 3, Zhongyang Rd, Hualien City 97002, Taiwan

${ }^{4}$ Department of Ophthalmology and Visual Science, Tzu Chi University,

Hualien, Taiwan

Full list of author information is available at the end of the article
} 


\section{Background}

Myopia is the most common refractive disorder, $[1,2]$ it is also one of the leading causes of visual impairment worldwide $[3,4]$. The prevalence of myopia varies geographically, for example, 30\% of the population in Australia [5] and North American [6], and up to 85\% of the East Asia population, especially in Taiwan $[7,8]$. Among myopic patients, high myopia is especially associated with increased risk of ocular comorbidities, $[9,10]$ increased socioeconomic burden, $[3,4,11,12]$ and compromised quality of life $[13,14]$. Therefore, the prevention of myopia progression is a major public health issue [8].

Many interventions have been developed to suppress the progression of myopia, including pharmaceutical agents (for example, atropine [15] and pirennzepine [16-18]), bifocal lenses [19, 20], multifocal lenses, [21, 22] aberration control spectacle lenses, [23] and soft and gas-permeable contact lenses [24-29]. Topical atropine reduces myopia progression and axial elongation in children in a doserelated manner. However, it has been reported to bring adverse effects, such as allergy, photophobia and a rebound phenomenon occur with higher doses [30, 31].

The concept of orthokeratology (OK) was first introduced in the 1950s by Wesley and Jessen as spectacle blur, a phenomenon describing corneal reshaping after wearing hard contact lenses. For its material was poor at oxygen permeation, making long-term wearing infeasible, orthokeratology was more of a novelty back then. In the 1970s, rigid gas permeable lenses improved comfort and safety by allowing more oxygen permeability. However, the lenses still remained incapable of effectively correcting myopia until the first reverse geometry lens designed by Richard Wlodyga introduced in 1989, which improved lens centration and myopia correction from -1 diopters (D) to $-1.7 \mathrm{D}$. Up to the present, improvement of orthokeratology mainly involves using higher Dk lens material, different reverse geometry lens designs, and advances in corneal topography [32].

By the reverse geometry design of orthokeratology lens, the lens molds the cornea of a myopic eye into plateau shape. These orthokeratology lenses have much flatter central base curve than the secondary curve, thus create positive pushing pressure against the central cornea and negative pulling pressure against the midperipheral cornea, redistributing the epithelial cells to the mid-periphery while flattening the central cornea via a thinning of the epithelial layer. Through plateaushaped cornea, light would be refracted simultaneously onto the mid-peripheral retina and macula, leaving the peripheral retina with relative myopic defocus [33-35]. Hyperopic peripheral defocus on the contrary, often found in myopic children, is believed to encourage eye growth. Manipulation of peripheral defocus toward myopia is hypothesized to stabilize eye growth and reduce myopia progression. Several studies reported its efficacy for myopia control by slowing axial elongation of the eyeball [36-38] and has been confirmed in a twoyear randomized clinical trial [39].

Somehow, there is limited publication with follow-up longer than 3 years. A five-year prospective study that assessed the efficacy of OK showed a reduced rate of manifest refraction progression in OK-wearing eyes in the first 3 year of treatment [40]. Two retrospective studies comparing children wearing $\mathrm{OK}$ lenses with single-vision spectacles showed a reduced rate of manifest refraction progression in OK-wearing eyes over 7year and 8-year periods [41, 42].

This retrospective study aims to investigate whether overnight $\mathrm{OK}$ influences the progression rate of the manifest refractive error of myopic children in a longer follow-up period (up to 12 years), and to investigate the relationship between refractive changes and different baseline factors, including refraction error, wearing age and lens replacement frequency. In addition, this study collects long-term safety profile of overnight OK.

\section{Methods}

This is a retrospective study of sixty-six school-age children who visited a private ophthalmology clinic between January 1998 and December 2013, and received overnight OK correction. Sixty-four school-age children wearing spectacles were identified from the same practice, and 36 subjects whose baseline age and refractive error matched with those in the OK group were selected to form control group. A single practitioner (CJC) performed all examinations of the OK and spectacle wearers. This study was conducted in accordance with the tenets of the Declaration of Helsinki and approved by the Institutional Ethical Committee Review Board (REC No.: IRB103-17-B).

The subjects included in this study must meet the following criteria: 1) below 18 years of age 2) spherical refractive error less than -8.0 D 3) cylinder refractive error less than -3.0 D 4) distant best-corrected visual acuity (BCVA) better than 0 log minimum angle of resolution (logMAR) units (20/20) 5) follow-up period greater than 12 months. Subjects with underlying ocular disease such as retinopathy, prematurity, neonatal problems, history of genetic disease, neurodevelopment condition that might affect refractive development, or connective tissue disorders associated myopia were excluded from this study. Enrolled subjects could not have any amount of tropia by cover-uncover test at far $(4.0 \mathrm{~m})$ and near $(0.33 \mathrm{~m})$. All subjects underwent comprehensive examination including manifest refraction, cycloplegic refraction, uncorrected visual acuity (UCVA), best-corrected visual acuity, extraocular movements, intraocular pressure, slit-lamp examination and dilated fundoscopy at the first visit. Manifest refraction and cycloplegic refraction following instillation 
of two drops of $1 \%$ tropicamide were obtained with autorefractor (Speedy-K, Nikon, Tokyo, Japan). Snellen visual acuity chart was placed $6 \mathrm{~m}$ from the patient, and the white background of the chart was illuminated to $85 \pm$ $5 \mathrm{~cd} / \mathrm{m}^{2}$.

An accelerated OK reverse geometry design lens (Dreimlens'; Macro Vision Corp., Taiwan) was used in this study. The nominal central thickness of the lenses was $0.22 \mathrm{~mm}$ and the diameter was 10.4 to $11.2 \mathrm{~mm}$. The material used in the overnight OK was a fluorosilicone acrylate material with nominal oxygen permeability (Dk) of $127 \times 10^{-11} \mathrm{~cm}^{2} / \mathrm{s}(\mathrm{mL} \mathrm{O} / \mathrm{mL} \mathrm{mmHg})$. The same practitioner (CJC) fitted all the OK lenses using standardized fitting criterion.

After baseline examination, each patient underwent a 2$h$ daytime trial before the first night of lens wearing to confirm a successful orthokeratology contact lens fit. If an acceptable contact lens fit and fluorescein pattern were established, subjects were instructed to wear their contact lenses for at least 6-8 consecutive hours every night. The recommended daily cleaning protocol is first cleaning lens with Boston Advanced Cleaner and Conditioning Solutions (Bausch \& Lomb), then rinsing the lenses with a sterile, preserved saline solution.

Clinical follow-up interval was 2-3 months on average. In follow-up examinations, UCVA and findings of slit-lamp examination of each visit were collected. Autorefraction and keratometry were performed using the Speedy-K autorefractor, cycloplegic refractions were measured every year. To minimize any potential acuity regression, all the examinations were completed before $11 \mathrm{AM}$ within 2 hours after contact lens removal. All instruments were calibrated before study initiation.

Refraction over lens, BCVA and lens inspection to assess lens condition were also collected at each visit. Changes to manifest refractive error were calculated indirectly by determining the change in treatment curve (back optic zone radius, BOZR) required to maintain a refraction over lens (ROL) of plano. To ensure the OK eyes were not over-treated, all lenses were designed to have achieved a ROL of plano at baseline. If ROL of -0.5 $\mathrm{D}$ was found, this would suggest an increase in the myopia compared with baseline. Based on the fitting principles, the BOZR would require flattening $0.1 \mathrm{~mm}$ to restore a full refractive treatment [43]. The lenses were replaced every 1 to 2 years on average in order to maintain the wearing quality. Stable lens replacement frequency was defined as average replacement period less than 2 years.

We recorded and compared the change of visual acuity and the change in corneal curvature, manifest refractive error by ROL method at baseline and at the 1-year to 12year follow-up. The manifest refractive error was expressed as spherical and cylindrical power, or power vectors: $M$ represents the spherical equivalent of a refraction, J0 represents the astigmatism power vector associated with a horizontally oriented axis, J45 corresponds to the astigmatism power vector associated with an obliquely oriented axis in the $45^{\circ}$ meridian $[44,45]$. The data were expressed as frequencies, proportions, or means \pm standard error of the mean, depending on the characteristics of each item. Some basic statistical tests were performed to compare the difference between groups. An independent $t$ test was used to compare the means of continuous variables between different groups, whereas a paired $t$ test was used to compare change of measurement results for paired samples. A Chi-squared test or Fisher Exact test was used to examine differences with categorical variables. Since correlated data from paired-eyes and repeated measurements across time were collected in this longitudinal study, the generalized estimating equations (GEE) was adopted to compare the results during the years to evaluate the association between the outcome and risk factors [46]. Statistically significant differences were defined as $p<0.05$. All of the statistical analyses were performed using SPSS software version 17.0 (SPSS Inc., Chicago, IL, USA).

\section{Results}

Data of 203 eyes were derived from 66 OK subjects (31 males and 35 females) and 36 control subjects (22 males and 14 females). Baseline demographics and ocular characteristics were summarized in Table 1 , there were no significant differences between the control and OK groups. Their wearing ages ranged from 7 years to 16 years (mean \pm SE, $11.72 \pm 0.18$ years). The follow-up time ranged from 1 year to 12 years (mean \pm SE, $6.32 \pm$ 0.15 years). At baseline, their myopia ranged from -0.5 $\mathrm{D}$ to $-8.0 \mathrm{D}$ (mean $\pm \mathrm{SE},-3.70 \pm 0.12 \mathrm{D})$, and astigmatism ranged from $0 \mathrm{D}$ to $-3.0 \mathrm{D}$ (mean $\pm \mathrm{SE},-0.55 \pm$ $0.05 \mathrm{D}$ ). The quantities of $\mathrm{M}, \mathrm{J} 0$, and $\mathrm{J} 45$ were $-3.54 \pm$ $0.14,-0.26 \pm 0.03$, and $-0.03 \pm 0.01$ (mean $\pm \mathrm{SE}$ ) respectively. The overall trend of refractive error change per two-year period of OK and control groups were shown in Fig. 1. Compared with control group, OK group had a significantly $(p<0.001)$ lower refractive error change during the follow-up period. Furthermore, characteristics of OK group were classified into two subgroups according to its extent or range and were summarized in Table 2. About $70.2 \%$ of OK subjects started orthokeratology corrections at the age more than 10 years old. About $30.5 \%$ of OK subjects were with myopia more than $-5.0 \mathrm{D}$. About $11.2 \%$ of subjects were with astigmatism greater than or equal to $-1.5 \mathrm{D}$. About $82.4 \%$ of OK subjects were wearing with stable lens replacement frequency, which was defined as average replacement period less than 2 years. 
Table 1 Comparison of Demographics between OK Group and Control Group

\begin{tabular}{|c|c|c|c|c|}
\hline Variable & OK Group & Control Group & $P$-value & Total \\
\hline Number of eyes & 131 & 72 & & 203 \\
\hline Wearing Age & $11.65 \pm 0.24$ & $11.83 \pm 0.26$ & 0.628 & $11.72 \pm 0.18$ \\
\hline Gender & & & 0.058 & \\
\hline Male & $61(46.6 \%)$ & $44(61.1 \%)$ & & 105 (51.7\%) \\
\hline Female & $70(53.4 \%)$ & $28(38.9 \%)$ & & $98(48.3 \%)$ \\
\hline Sphere refraction (D) & $-3.82 \pm 0.14$ & $-3.48 \pm 0.20$ & 0.167 & $-3.70 \pm 0.12$ \\
\hline Cylinder refraction (D) & $-0.56 \pm 0.07$ & $-0.52 \pm 0.08$ & 0.683 & $-0.55 \pm 0.05$ \\
\hline M & $-3.54 \pm 0.14$ & $-3.22 \pm 0.19$ & & $-3.43 \pm 0.12$ \\
\hline J0 & $-0.26 \pm 0.03$ & $-0.25 \pm 0.04$ & & $-0.26 \pm 0.03$ \\
\hline$J 45$ & $-0.03 \pm 0.01$ & $0.03 \pm 0.01$ & & $-0.01 \pm 0.01$ \\
\hline Follow-up Time (years) & $6.20 \pm 0.21$ & $6.53 \pm 0.17$ & 0.291 & $6.32 \pm 0.15$ \\
\hline
\end{tabular}

Data are presented as $\mathrm{n}(\%)$ or mean \pm standard error of the mean

${ }^{*} p$-value $<0.05$ was considered statistically significant after test

$\mathrm{D}$, diopters; $\mathrm{M}$, the spherical equivalent; J0, the astigmatism power vector associated with a horizontally oriented axis; J45, the astigmatism power vector associated with an obliquely oriented axis in the $45^{\circ}$ meridian

Further analysis was performed for OK subjects to evaluate the progression rate of the manifest refractive changes and of the association between the manifest refractive changes and baseline factors. The effect of orthokeratology corrections on change of corneal curvature per two-year period decreased gradually in the follow-up years (Table 3). There was decreased follow-up rate after the first 4 years, around only $14 \%$ of subjects remained in the study after 8 years. Therefore, statistical analysis of evaluating the association between the manifest refractive change and risk factors was performed for both follow up periods, including first 8 years and total 12 years. According to the analysis results of GEE model (Tables 4 and 5), astigmatism greater than or equal to $-1.5 \mathrm{D}$ and greater J0 (more negative) were found to be associated with increased change of refractive error during follow-up years. The trends of refractive error change per two-year period for different wearing age groups all showed that the change increased gradually in the first few years then started to decrease afterwards (Fig. 2a). Subjects with wearing age more than 10 years old tend to have larger refractive error change than those with wearing age less than 10 years old during first 4 years, but the overall difference between two groups was not significant in GEE model. The trends of refractive error change per two-year period for different initial myopic refraction groups all showed that the change increased gradually in the first few years then started to decrease afterwards (Fig. 2b).

\section{Trend of refractive error change by case and control groups}

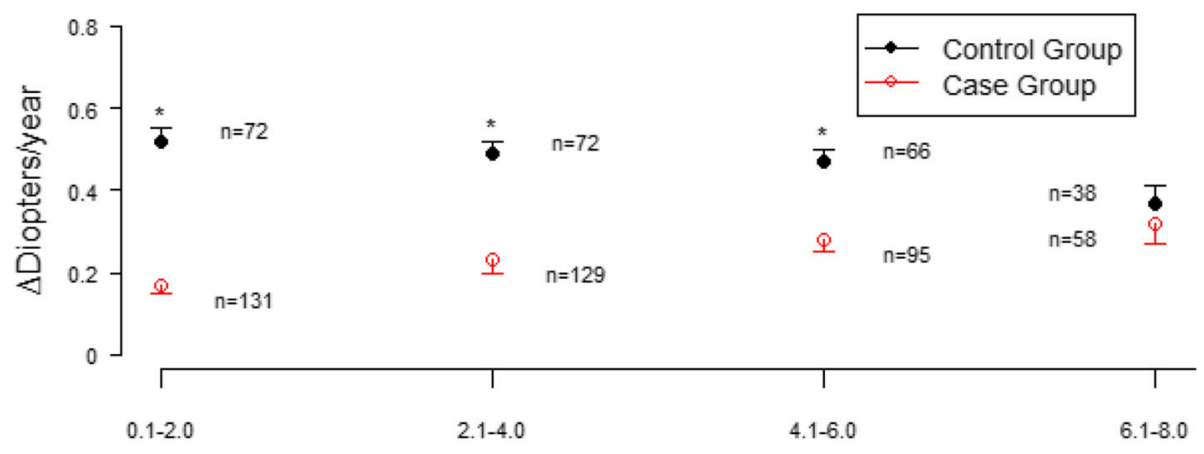

Follow-up Periods (Years)

Fig. 1 Trend of refractive error change. The trend of refractive error change during year 0 to year 2 for OK and control groups were $0.17 \pm 0.02 \mathrm{D}$ and $0.52 \pm 0.03 \mathrm{D}$ (mean $\pm \mathrm{SE}$ ), respectfully. The trend of refractive error change during year 2 to year 4 for OK and control groups were $0.23 \pm 0.03 \mathrm{D}$ and $0.50 \pm 0.03 \mathrm{D}$ (mean $\pm \mathrm{SE}$ ), respectfully. The trend of refractive error change during year 4 to year 6 for OK and control groups were $0.28 \pm 0.03 \mathrm{D}$ and $0.47 \pm 0.03 \mathrm{D}$ (mean $\pm \mathrm{SE}$ ), respectfully. The trend of refractive error change during year 6 to year 8 for OK and control groups were $0.32 \pm 0.05 \mathrm{D}$ and $0.37 \pm 0.04 \mathrm{D}$ (mean $\pm \mathrm{SE}$ ), respectfully. Asterisk $\left(^{*}\right)$ denotes statistically significant difference between OK and control groups 
Table 2 Baseline Characteristics and Treatment Parameters of 66 OK subjects

\begin{tabular}{ll}
\hline Variable & $\mathrm{n}(\%)$ or mean \pm S.E. \\
\hline Number of eyes & 131 \\
Gender & \\
Male & $61(46.6 \%)$ \\
Female & $70(53.4 \%)$ \\
Age & $11.65 \pm 0.24$ \\
$\quad<=10$ years-old & $39(29.8 \%)$ \\
$>10$ years-old & $92(70.2 \%)$ \\
Ocular sphere refraction (D) & $-3.82 \pm 0.14$ \\
$<-5 D$ & $91(69.5 \%)$ \\
$>=-5 D$ & $40(30.5 \%)$ \\
Ocular cylinder refraction (D) & $-0.56 \pm 0.07$ \\
$<-1.5 D$ & $117(89.3 \%)$ \\
$>=1.5 D$ & $14(10.7 \%)$ \\
M & $-3.54 \pm 0.14$ \\
J0 & $-0.26 \pm 0.03$ \\
J45 & $-0.03 \pm 0.01$ \\
Keratometry value (D) & $43.64 \pm 0.11$ \\
Follow-up time (years) & $6.20 \pm 0.21$ \\
Regular lens replacement & \\
No & $23(17.6 \%)$ \\
\hline Yes & $108(82.4 \%)$ \\
\hline
\end{tabular}

Data are presented as $\mathrm{n}(\%)$ or mean \pm standard error of the mean $D$, diopters; $M$, the spherical equivalent; J0, the astigmatism power vector associated with a horizontally oriented axis; $\mathrm{J45}$, the astigmatism power vector associated with an obliquely oriented axis in the $45^{\circ}$ meridian

The overall difference between two groups was also not significant in GEE model. The trends of refractive error change per two-year period for different initial astigmatism groups showed significant difference (Fig. 2c). Subjects with astigmatism greater than or equal to $-1.5 \mathrm{D}$ tend to have larger increase of refractive error change during follow-up years $(p=0.007$, Fig. $2 c$ ). The overall difference between two groups was

Table 3 The effect of orthokeratology corrections at different periods over 12 years follow-up

\begin{tabular}{lllllll}
\hline Item & $\begin{array}{l}\text { 1st to } \\
\text { 2nd year }\end{array}$ & $\begin{array}{l}\text { 3rd to } \\
\text { 4th year }\end{array}$ & $\begin{array}{l}\text { 5th to } \\
\text { 6th year }\end{array}$ & $\begin{array}{l}\text { 7th to } \\
\text { 8th year }\end{array}$ & $\begin{array}{l}\text { 9th to } \\
\text { 10th year }\end{array}$ & $\begin{array}{l}\text { 11th to } \\
12 \text { th year }\end{array}$ \\
\hline $\begin{array}{l}\text { Number } \\
\text { of eyes }\end{array}$ & 131 & 129 & 95 & 58 & 18 & 8 \\
$\begin{array}{l}\text { Change } \\
\text { of }\end{array}$ & $0.17 \pm$ & $0.23 \pm$ & $0.28 \pm$ & $0.32 \pm$ & $0.23 \pm$ & $0.06 \pm$ \\
refractive & 0.02 & 0.03 & 0.03 & 0.05 & 0.07 & 0.04 \\
error & & & & & & \\
$\begin{array}{l}\text { Change } \\
\text { of corneal } \\
\text { curvature }\end{array}$ & 0.08 & 0.09 & 0.11 & 0.16 & 0.30 & 0.69 \\
\hline
\end{tabular}

Data are presented as $\mathrm{n}$ or mean \pm standard error of the mean
Table 4 GEE Model predicting change of refractive error over 8 years follow-up. $(n=131)$

\begin{tabular}{lllll}
\hline Predictor & $\beta$ & S.E. & $Z$ & $p$ value \\
\hline A & & & & \\
Intercept & -0.039 & 0.105 & -0.37 & 0.711 \\
Wearing Age(>10 y/o vs. $<=10$ y/o) & 0.063 & 0.051 & 1.24 & 0.215 \\
Stable Change(Yes vs. No) & -0.014 & 0.010 & -1.43 & 0.151 \\
Initial D(>=-5D vs. <-5D) & -0.012 & 0.054 & -0.22 & 0.829 \\
Initial A(>=-1.5D vs. $<-1.5 D)$ & 0.181 & 0.070 & 2.60 & $0.009^{*}$ \\
B & & & & \\
Intercept & -0.039 & 0.105 & -0.37 & 0.711 \\
Wearing Age (>10 y/o vs. <=10 y/o) & 0.063 & 0.051 & 1.24 & 0.215 \\
Stable Change (Yes vs. No) & -0.014 & 0.010 & -1.43 & 0.151 \\
M & -0.012 & 0.010 & -1.14 & 0.255 \\
J0 & -0.189 & 0.051 & -3.72 & $<0.001^{*}$ \\
J45 & -0.076 & 0.099 & -0.77 & 0.444 \\
\hline
\end{tabular}

Abbreviation: GEE, generalized estimating equations

${ }^{*} \mathrm{p}$-value $<0.05$ was considered statistically significant after test

$D$, diopters; $M$, the spherical equivalent; $J 0$, the astigmatism power vector associated with a horizontally oriented axis; $\mathrm{J45}$, the astigmatism power vector associated with an obliquely oriented axis in the $45^{\circ}$ meridian

significant in both GEE models. The trends of refractive error change per two-year period for stable and unstable lens replacement groups showed that the change increased gradually in the first few years then started to decrease afterwards (Fig. 2d). The overall difference between two groups was not significant in GEE model.

Table 5 GEE Model predicting change of refractive error over 12 years follow-up. $(n=131)$

\begin{tabular}{lllll}
\hline Predictor & $\beta$ & S.E. & $Z$ & $p$ value \\
\hline a & & & & \\
Intercept & -0.010 & 0.091 & -0.11 & 0.916 \\
Wearing Age(>10 y/o vs. $<=10$ y/o) & 0.058 & 0.050 & 1.17 & 0.240 \\
Stable Change(Yes vs. No) & -0.013 & 0.010 & -1.38 & 0.167 \\
Initial D(>=-5D vs. <-5D) & -0.007 & 0.053 & -0.13 & 0.898 \\
Initial A(>=-1.5D vs. <-1.5D) & 0.153 & 0.056 & 2.75 & $0.006^{*}$ \\
b & & & & \\
Intercept & -0.010 & 0.091 & -0.11 & 0.916 \\
Wearing Age (>10 y/o vs. <=10 y/o) & 0.058 & 0.050 & 1.17 & 0.240 \\
Stable Change (Yes vs. No) & -0.013 & 0.010 & -1.38 & 0.167 \\
M & -0.013 & 0.010 & -1.38 & 0.169 \\
J0 & -0.182 & 0.045 & -4.02 & $<0.001^{*}$ \\
J45 & -0.045 & 0.102 & -0.44 & 0.659 \\
\hline
\end{tabular}

Abbreviation: GEE, generalized estimating equations

${ }^{*} p$-value $<0.05$ was considered statistically significant after test

$\mathrm{D}$, diopters; $\mathrm{M}$, the spherical equivalent; $\mathrm{J}$, the astigmatism power vector associated with a horizontally oriented axis; $\mathrm{J45}$, the astigmatism power vector associated with an obliquely oriented axis in the $45^{\circ}$ meridian 


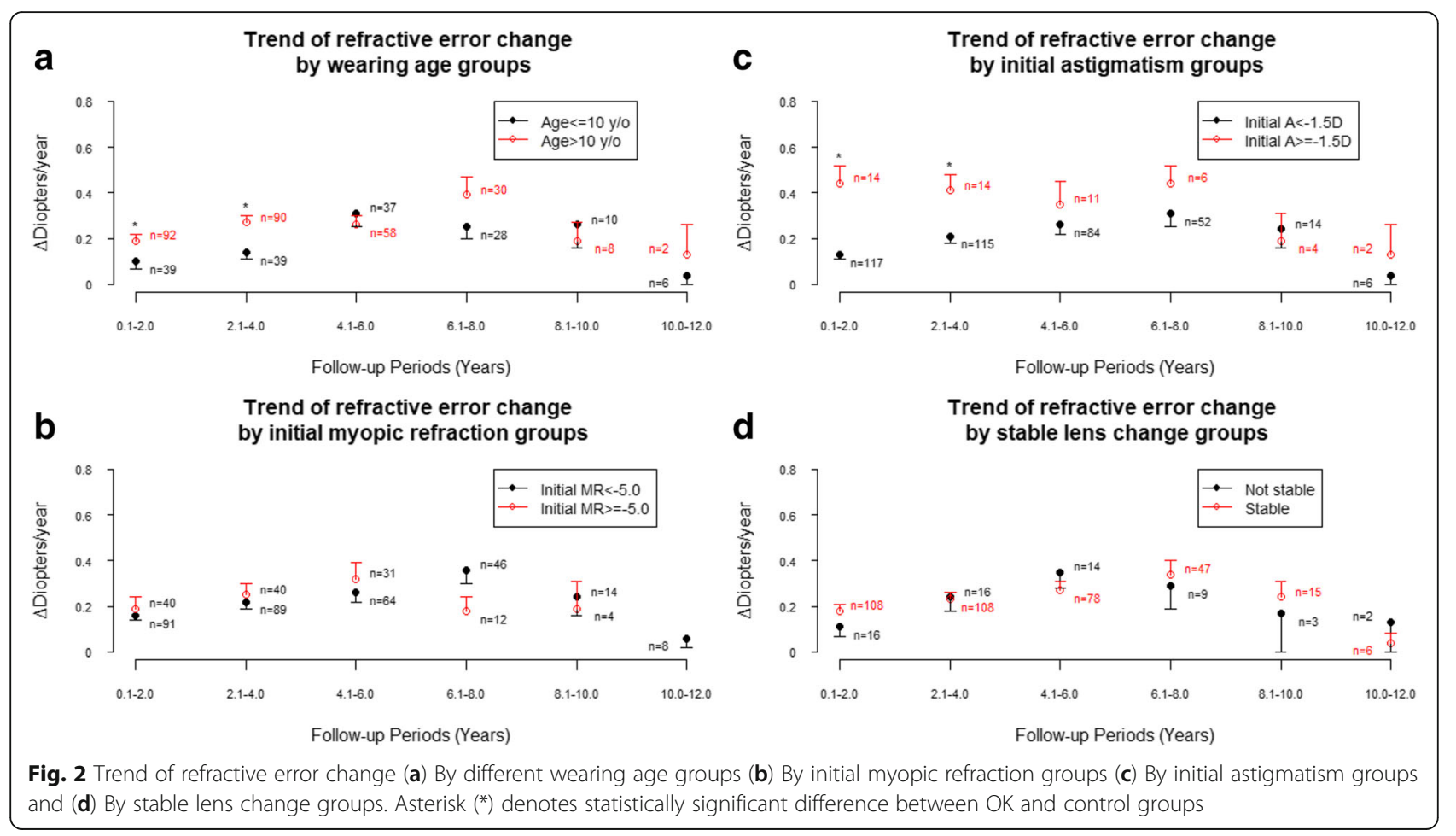

During the study period, mild superficial punctate keratopathy was observed in 8 subjects and mild corneal erosion was noted in 2 subjects. But these events recovered completely after discontinuation of lens wear for 1 to 2 weeks, and lens re-design in one subject. No other severe complications, such as corneal ulcer, were found in these OK users.

\section{Discussion}

In this retrospective study with long term follow-up period (up to 12 years) conducted to investigate whether where overnight OK influences the progression of manifest refractive error in myopic children, OK group had a significantly $(p<0.001)$ lower refractive error change during the follow-up period. The refractive error change of OK group was around 0.2 to $\sim 0.3$ diopters per /year, while those wearing single-vision glasses had 0.4 to $\sim 0.5$ diopters per /year. Reviewing prior publications, published literature, the rate of myopia progression in our control group is comparable to other studies regarding myopic control using by orthokeratology [35-38]. Although previous reports demonstrate that overnight $\mathrm{OK}$ can retard the rate of myopia progression, there is limited published data beyond 3 years of follow-up. Our results revealed myopia control effect by orthokeratology could up to 8 years, with lessening the differences between the two groups gradually. ROMIO study [39] reported a time dependent apparent reduced efficacy on myopic control using $\mathrm{OK}$, which was also observed in Hiraoka et al.'s results [40]. In their opinion, the reduction resulted from the gradual slowing of myopic progression in the control group with age, which was confirmed as a natural process, instead of reduced OK efficacy. This finding was also reported in prior studies [36-39, 47]. In a five-year prospective study by Hiaroka et al., they reported no additional beneficial effect for retarding myopia progression using orthokeratology after 3 years of lens wear [45]. However, children tend to have slower myopia progression with age, which was confirmed by previous studies. In meta-analysis by Donovan et al., myopia progression was faster in younger children, with greatest change of myopia in Chinese children reported ranging in age from 9 to 11 years [46]. Our results showed better myopia control by orthokeratology in the first 8 years of the study period, with the differences between the two groups narrowing gradually. The reduced myopic control effect may due to decreasing progression as age increased. This finding was also reported in prior studies [35-38].

Our study utilized standard lens design (spherical 4zone lens), this limited the result applied only to children with low-to-moderate myopia and low astigmatism and who could achieve satisfactory orthokeratology response. Nowadays, different lens designs aiming to improve the performance of orthokeratology lenses became available, such as toric orthokeratology designs [40, 47]. It is expected orthokeratology for myopic control now applicable to a wider range of population in terms of children with higher degrees of myopia and astigmatism, thereby allowing more children to benefit from the myopic control treatment using orthokeratology. Many 
studies have been conducted to test if the performance of orthokeratology lenses to reduce myopia regression improved by new lens designs [48].

In our analysis of the relationship between refractive power change and different baseline refraction error, wearing age and lens replacement frequency, to evaluate the effect of orthokeratology corrections and its association between characteristics, only initial astigmatism power was found to be associated with change of refractive error progression during 12 years of follow -up years. The higher degrees of initial astigmatism, the greater progression of refractive error overmyopia refraction power with time. As with-the-rule astigmatism is the most common astigmatism in childhood, we found the absolute value of J0 was greater than $\mathrm{J} 45$ in both OK and control groups. Both high astigmatism $(>-1.5 \mathrm{D})$ and greater J0 (more negative) were found to be associated with increased change of refractive error during follow-up years. We hypothesize the lack of toric orthokeratology lens for high astigmatism may lead to incomplete myopic defocus of peripheral retina, thereby offset the effect of retarding myopia progression.The lack of toric orthokeratology lens for high astigmatism may led to incomplete myopic defocus of peripheral retina, thereby offset the effect of retarding myopia progression. In addition, the disparity of case number between children with high astigmatism power and those with less astigmatism power, may have made the statistical result unstable.

One limitation of our study is that myopia progression was measured by change of refraction over lens but not axial length, thus the reported differences in myopic refractive change was not necessarily correlated to real differences in ocular growth. Somehow, the same approach was used in Downie et al.'s study with reported advantages of its simple and all-embracing nature [42]. Thus, we confer the finding of change of refraction over lens in this study is related to myopic progression, not just a temporary effect. There is another limitation that tropicamide was used as cycloplegic agent instead of cyclopentolate. A previous study has shown $1 \%$ tropicamide is an effective cycloplegic agent for myopic children [48]. Cycloplegic refraction using $1 \%$ tropicamide is a proven method and was used in published study [49]. We also acknowledge the retrospective design of this study might carry the potential bias. Moreover, the disparity of case number between high astigmatism and low astigmatism may have made the statistical result unstable [41]. Besides, the retrospective design of this study might also carry the potential for both practitioner and investigator bias as neither the examiner nor the patient were masked to the treatment groups. Only standard-design lens (spherical 4-zone lens) was available during our study period limited the result applied only to children with low-to-moderate myopia and low astigmatism and who could achieve satisfactory orthokeratology response. Nowadays, different lens designs aiming to improve the performance of orthokeratology lenses became available, such as toric orthokeratology designs $[50,51]$. Orthokeratology now is more applicable to a wider range of population with higher degrees of myopia and astigmatism. Besides, the optimal treatment duration and ideal starting age remain unclear. Our further clinical studies will try to clarify these issues.

\section{Conclusions}

In conclusion, our study showed that OK treatment was effective in slowing progression of myopic refractive error over a twelve-year treatment period and demonstrated a clinically acceptable safety profile in a population of patients aged seven to sixteen years. Initial astigmatism power was the essential influential factor of change in refractive error during follow-up years. Initial wearing age, initial myopic power and lens replacement were found to have no effect on progression of myopic refractive error.

\section{Abbreviations}

BCVA: Best-corrected visual acuity; D: Diopters; GEE: Generalized estimating eqs.; J0: the astigmatism power vector associated with a horizontally oriented axis; J45: the astigmatism power vector associated with an obliquely oriented axis in the $45^{\circ}$ meridian; logMAR: log minimum angle of resolution; M: the spherical equivalent; OK: Orthokeratology; ROL: Refraction over lens; UCVA: Uncorrected visual acuity

\section{Acknowledgements}

Not applicable.

Funding

None.

\section{Availability of data and materials}

The datasets used and analyzed during the current study are available from the corresponding author on reasonable request.

\section{Authors' contributions}

YCL participated in analysis and interpretation of statistics, manuscript writing. JHW participated in acquisition of data, analysis and interpretation of statistics, figure preparation. CJC participated in acquisition of data, study concept and design, revision of the manuscript for important intellectual content. All authors read and approved the final manuscript.

\section{Ethics approval and consent to participate}

This study was conducted in accordance with the tenets of the Declaration of Helsinki and approved by the Institutional Ethical Committee Review Board of Buddhist Tzu Chi General Hospital, Hualien, Taiwan (REC No: IRB103-17-B). We confirmed that all written consents were obtained from participants. For those participants under the age of 16 , the consent forms were signed by the parents on behalf of those participants.

\section{Consent for publication}

Not applicable.

\section{Competing interests}

The authors declare that they have no competing interests.

\section{Publisher's Note}

Springer Nature remains neutral with regard to jurisdictional claims in published maps and institutional affiliations. 


\section{Author details}

'Department of Ophthalmology, Buddhist Tzu Chi General Hospital, No. 707, Sec. 3, Zhongyang Rd, Hualien City 97002, Taiwan. ${ }^{2}$ Department of Medical Research, Buddhist Tzu Chi General Hospital, Hualien, Taiwan. ${ }^{3}$ Institute of Medical Sciences, Tzu Chi University, Hualien, Taiwan. ${ }^{4}$ Department of Ophthalmology and Visual Science, Tzu Chi University, Hualien, Taiwan.

\section{Received: 19 June 2017 Accepted: 29 November 2017}

\section{Published online: 08 December 2017}

\section{References}

1. Angle J, Wissmann DA. The epidemiology of myopia. Am J Epidemiol. 1980; 111:220-8.

2. Javitt JC, Chiang YP. The socio-economic aspects of laser refractive surgery. Arch Ophthalmol. 1994;112:1526-30.

3. Dandona R, Dandona L. Refractive error blindness. Bull World Health Organ. 2001;79:237-43

4. McCarty CA. Uncorrected refractive error. Br J Ophthalmol. 2006;90:521-2.

5. Wensor M, McCarty CA, Taylor HR Prevalence and risk factors of myopia in Victoria, Austalia Arch Oohthalmol 1999,117658-63.

6. Vitale $S$, Ellwein $L$, Cotch MF, et al. Prevalence of refractive error in the United States, 1999-2004. Arch Ophthalmol. 2008:126:1111-9.

7. Lin LL, Shih YF, Hsiao CK, et al. Epidemiologic study of the prevalence and severity of myopia among school children in Taiwan in 2000. J Formos Med Assoc. 2001;100:684-91.

8. Wang TJ, Chiang $\mathrm{TH}$, Wang $\mathrm{TH}$, et al. Changes of the ocular refraction among freshmen in National Taiwan University between 1988 and 2005. Eye(Lond). 2009;23:1168-9.

9. Liu HH, Xu L, Wang YX, et al. Prevalence and progression of myopic retinopathy in Chinese adults: the Beijing eye study. Ophthalmology. 2010;117:1763-8.

10. Yannuzzi LA, Sorenson JA, Sobel RS, et al. Risk-factors for idiopathic rhegmatogenous retinal-detachment. Am J Epidemiol. 1993;137:749-57.

11. Bourne RR, Dineen BP, Ali SM, et al. Prevalence of refractive error in Bangladeshi adults: results of the National Blindness and low vision survey of Bangladesh. Ophthalmology. 2004;111:1150-60.

12. Wong TY, Loon SC, Saw SM. The epidemiology of age related eye diseases in Asia. Br J Ophthalmol. 2006;90:506-11.

13. Vitale $\mathrm{S}$, Schein $\mathrm{OD}$, Meinert $\mathrm{CL}$, et al. The refractive status and vision profile: a questionnaire to measure vision-related quality of life in persons with refractive error. Ophthalmology. 2000;107:1529-39.

14. Rose K, Harper R, Tromans C, et al. Quality of life in myopia. Br J Ophthalmol. 2000;84:1031-4.

15. Chia $\mathrm{C}$, Chua $\mathrm{W}-\mathrm{H}$, Cheung $\mathrm{Y}-\mathrm{B}$, et al. Atropine for the treatment of childhood myopia: safety and efficacy of $0.5 \%, 0.1 \%$ and $0.01 \%$ doses (atropine for the treatment of myopia 2). Ophthalmology. 2012;119:347-54

16. Siatkowski RM, Cotter SA, Crockett RS, et al. Two-year multicenter, randomized, double-masked, placebo-controlled, parallel safety and efficacy study of $2 \%$ pirenzepine ophthalmic gel in children with myopia. J AAPOS. 2008:12:332-9.

17. Tan DT, Lam DS, Chua WH, et al. One-year multicenter, double-masked, placebo-controlled, parallel safety and efficacy study of 2\% pirenzepine ophthalmic gel in children with myopia. Ophthalmology. 2005;112:84-91.

18. Siatkowski RM, Cotter S, Miller JM, et al. Safety and efficacy of $2 \%$ pirenzepine ophthalmic gel in children with myopia: a 1-year, multicenter, double-masked, placebo-controlled parallel study. Arch Ophthalmol. 2004; 122:1667-74.

19. Fulk GW, Cyert LA, Parker DE. A randomized trial of the effect of singlevision vs. bifocal lenses on myopia progression in children with esophoria. Optom Vis Sci. 2000;77:395-401.

20. Cheng D, Schmid KL, Woo GC, et al. Randomized trial effect of bifocal and prismatic bifocal spectacles on myopia progression. Arch Ophthalmol. 2010; 128:12-9.

21. Leung JT, Brown B. Progression of myopia in Hong Kong Chinese schoolchildren is slowed by wearing progressive lenses. Optom Vis Sci. 1999;76:346-54.

22. Yang Z, Lan W, Ge J, et al. The effectiveness of progressive addition lenses on the progression of myopia in Chinese children. Ophthalmic Physiol Opt. 2009;29:41-8.

23. Sankaridurg P, Donovan L, Varnas $S$, et al. Spectacle lenses designed to reduce progression of myopia: 12-month results. Optom Vis Sci. 2010;87: $631-41$.
24. Aller TA, Wildsoet C. Bifocal soft contact lenses as a possible myopia control treatment: a case report involving identical twins. Clin Exp Optom. 2008:91:394-9.

25. Stone J. The possible influence of contact lenses on myopia. Br J Physiol Opt. 1976:31:89-114.

26. Grosvenor T, Perrigin J, Perrigin D, et al. Use of silicone-acrylate contact lenses for the control of myopia. Results after two years of lens wear. Optom Vis Sci. 1989;66:41-7.

27. Perrigin J, Perrigin D, Quintero S, et al. Silicone acrylate contact lenses for myopia control: 3-year results. Optom Vis Sci. 1990;67:764-9.

28. Katz J, Schein OD, Levy B, et al. A randomized trial of rigid gas permeable contact lenses to reduce progression of children's myopia. Am J Ophthalmol. 2003;136:82-90.

29. Walline JJ, Jones LA, Mutti DO, et al. A randomized trial of the effects of rigid contact lenses on myopia progression. Arch Ophthalmol. 2004;122: 1760-6.

30. Cooper J, Schulman E, Jamal N. Current status on the development and treatment of myopia. Optometry. 2012;83:179-99.

31. Gwiazda J. Treatment options for myopia. Optom Vis Sci. 2009;86:624-8.

32. Swarbrick HA. Orthokeratology review and update. Clin Exp Optom. 2006;89: 124-43.

33. Nichols JJ, Marsich MM, Nguyen M, et al. Overnight orthokeratology. Optom Vis Sci. 2000:77:252-9.

34. Caroline PJ. Contemporary orthokeratology. Cont Lens Anterior Eye. 2001;24: $41-6$

35. Cheung SW, Cho P, Chui WS, et al. Refractive error and visual acuity changes in orthokeratology patients. Optom Vis Sci. 2007:84:410-6.

36. Cho $P$, Cheung SW, Edwards $M$. The longitudinal orthokeratology research in children (LORIC) in Hong Kong: a pilot study on refractive changes and myopic control. Curr Eye Res. 2005;30:71-80.

37. Walline JJ, Jones LA, Sinnott LT. Corneal reshaping and myopia progression. Br J Ophthalmol. 2009:93:1181-5.

38. Kakita T, Hiraoka T, Oshika T. Influence of overnight orthokeratology on axial elongation in childhood myopia. Invest Ophthalmol Vis Sci. 2011:52:2170-4.

39. Cho P, Cheung SW. Retardation of myopia in Orthokeratology (ROMIO) study: a 2-year randomized clinical trial. Invest Ophthalmol Vis Sci. 2012;53: 7077-85.

40. Hiraoka T, Kakita T, Okamoto F, et al. Long-term effect of overnight orthokeratology on axial length elongation in childhood myopia: a 5-year follow-up study. Invest Ophthalmol Vis Sci. 2012:53:3913-9.

41. Mok AK-H, Chung CS-T. Seven-year retrospective analysis of the myopic control effect of orthokeratology in children: a pilot study. Clin Optom. 2011;3:1-4.

42. Downie LE, Lowe R. Corneal reshaping influences myopic prescription stability (CRIMPS): an analysis of the effect of orthokeratology on childhood myopic refractive stability. Eye \& Contact Lens: Science \& Clinical Practice. 2013:39:303-10

43. Taiwan Macro Vision Corp. Professional fitting guide of orthokeratology; 2013. p. pp7-14.

44. Thibos LN, Wheeler W, Horner D. Power vectors: an application of Fourier analysis to the description and statistical analysis of refractive error. Optom Vis Sci. 1997;74:367-75.

45. Miller JM. Clinical applications of power vectors. Optom Vis Sci. 2009:86: 599-602.

46. Fan $\mathrm{Q}$, Teo YY, Saw SM. Application of advanced statistics in ophthalmology. Invest Ophthalmol Vis Sci. 2011:52:6059-65.

47. Donovan L, Sankaridurg P, Ho A, Naduvilath T, et al. Myopia progression rates in urban children wearing single-vision spectacles. Optom Vis Sci. 2012;89:27-32

48. Manny RE, Hussein M, Scheiman M, et al. Tropicamide (1\%): an effective cycloplegic agent for myopic children. Invest Ophthalmol Vis Sci. 2001;42: 1728-35.

49. Marsh-Tootle WL, Dong LM, Hyman L, et al. Myopia Progression in Children Wearing Spectacles vs. Switching to Contact Lenses. Optom Vis Sci. 2009; Epub ahead of print

50. Chen C, Cheung SW, Cho P, et al. Myopia control using toric orthokeratology (TO-SEE study). Invest Ophthalmol Vis Sci. 2013;54:6510-7.

51. Paune J, Cardona G, Quevedo L, et al. Toric double tear reservoir contact lens in orthokeratology for astigmatism. Eye Contact Lens. 2012;38:245-51. 